\title{
Ações de enfermagem para a prevenção da extubação acidental
}

\author{
Nursing interventions for the prevention of accidental extubation \\ Intervenciones de enfermería para la prevención de la extubación accidental
}

\section{Théia Maria Forny Wanderley Castellões', Lolita Dopico da Silva'}

'Universidade do Estado do Rio de Janeiro. Faculdade de Enfermagem. Rio de Janeiro, RJ

Submissão: 29/08/2008

Aprovação: 2 l/06/2009

\section{RESUMO}

Resumo: Objetivo foi apresentar os resultados parciais da incidência da extubação acidental associada ao cuidado de enfermagem. Método: estudo observacional retrospectivo, de intervenção prospectiva, medindo antes e depois da implantação de um guia as extubações acidentais A população de 142 pacientes,sendo 72 pacientes na fase retrospectiva e 70 na fase prospectiva,totalizando 3771 dias de ventilação. Dados coletados de prontuários.Resultados: 52,78\% usavam tubo traqueal e 62\% tinham entre 71 e 90 anos com média de dias de ventilação de 26,5 dias,. Ocorreram seis $(3,27)$ extubações antes e duas $(1,03)$ após a implantação do guia.Conclusões, houve diminuição da incidência da extubação acidental no período estudado, mas não se pode atribuir ao guia esta diferença pois são necessários outros estudos.

Descritores: Enfermagem; Cuidados intensivos; Ventilação mecânica.

\begin{abstract}
The objective was to analyze results of the incidence of accidental extubation associated with nursing care. Method: Retrospective observational study, the intervention ahead, measured before and after the deployment of a guide extubations accidental The population of 142 patients, 72 patients in the retrospective phase and 70 in phase ahead, totaling 3771 days of ventilation. Data collected from prontuários. Results: $52.78 \%$ used tracheal tube and $62 \%$ were between 71 and 90 years with an average of days of ventilation of 26.5 days,. There were six (3.27) extubations before and two (1.03) after the deployment of guia. Conclusion, there was a decrease in the incidence of accidental extubation in the period studied, but one can not attribute this difference to the guide because other studies are needed.
\end{abstract}

Descriptors: Nursing; Intensive care; Respiration, artificial.

\section{RESUMEN}

Objetivo: presentar los resultados parciales de la incidencia de la extubación accidental relacionada al cuidado de Enfermería.Método: estudio observacional retrospectivo de intervención prospectiva, midiendo antes y después de un protocolo las extubaciones accidentales. LA población fue de 142 pacientes, siendo 72 en el periodo retrospectivo y 70 en el prospectivo, haciendo 377 I días de ventilación. Datos colectados de prontuarios. Resultados : 52,78\% usavam tubo traqueal y 62\% tenian entre 71 y 90 anos com 26,5 média de dias de ventilación. Ocurrieron seis $(3,27)$ extubaciones antes y dos $(1,03)$ después de la implantación del protocolo. Conclusiones, hubo disminución de la incidencia de la extubación accidental en el período estudiado, pero no se pode atribuir al guía esta diferenza pues son necesarios otros estudios.

Descriptores: Enfermería; Cuidados intensivos; Respiración artificial. 


\section{INTRODUÇÃO}

A extubação acidental ou não planejada, é entendida como a retirada inadvertida e não intencional do dispositivo ventilatório ${ }^{1} \mathrm{e}$ caracterizada como um evento adverso do cuidado, Quando ocorre ocasiona aumento da morbidade e mortalidade, e, estando relacionada ao cuidado de enfermagem exige reflexão sobre as causas Que levaram à sua ocorrência ${ }^{(1)}$.

Quando uma extubação acidental ocorre há conseQüências para o paciente em diversos aspectos. Sempre se torna necessário a reintubação e há um aumento do tempo de ventilação mecânica Que gera maior tempo de internação. ConseQüentemente aumenta o risco de hipoxemia, atelectasia, pneumonia associada a ventilação mecânica (PAV), lesão em traQuéia, instabilidade hemodinâmica, parada cardíaca e às vezes até a morte ${ }^{(1)}$.

Estudos $^{(2)}$ destacam como outra conseQüência a pneumonia secundária, a dispnéia, trauma de via aérea, edema e dificuldade na reintubação. Por outro lado, dentre os fatores mais citados na literatura para a extubação não planejada, destacam-se a contenção física, o nível de sedação e atividade, e o status mental do paciente no período da extubação. O fator mais comumente associado com a extubação não acidental na UTI é a técnica de fixação do TOT ${ }^{(3-5)}$.

Neste estudo, a extubação não planejada está focalizada na extubação acidental associada ao cuidado de enfermagem. Considerando os aspectos clínicos de morbidade associados a extubação acidental citados anteriormente, a equipe de enfermagem de uma UTI, deve se esforçar ao máximo para evitar a ocorrência deste evento nos pacientes ali internados.

Através de um banco de dados existentes na UTI do Hospital Pró-Cardíaco, identificou-se Que as extubações acidentais Quando ocorriam associadas ao cuidado de enfermagem eram nos momentos de banho no leito, mudança de decúbito, troca de fixação e transporte interno do paciente. Com estes dados percebeu-se a necessidade de se intervir nos momentos em Que o cuidado de enfermagem poderia causar a extubação acidental. A partir daí, surgiu à idéia de elaborar um guia para a prevenção da extubação.

Este artigo tem como objetivo apresentar os resultados parciais de uma pesquisa relacionada à incidência da extubação acidental, após a implantação de um guia preventivo da extubação. O guia fundamentou-se nos Quatro momentos do cuidado de enfermagem onde se registravam as extubações: banho no leito, troca da fixação, mudança de decúbito, transporte do paciente.

$\mathrm{O}$ cuidado de enfermagem deve gerar segurança ao paciente $\mathrm{e}$ sua família deve sentir confiança na equipe multiprofissional, Que precisa ser efetiva para contribuir com a evolução do paciente, prevenindo suas complicações, reduzindo o tempo de estada na Unidade de Terapia Intensiva e com isso o custo pessoal e familiar de uma internação.

\section{MÉTODO}

Estudo observacional retrospectivo, de intervenção prospectiva. Consistiu em medir antes e depois da implantação do guia as ocorrências de extubações acidentais associadas ao cuidado enfermagem. O campo de peseuisa foi a Unidade de Terapia Intensiva (UTI) do Hospital Pró-Cardíaco, com capacidade de Quinze leitos. A Unidade de Terapia Intensiva é dividida em I e II, sendo as duas localizadas no primeiro andar do hospital, não estando interligadas. Treze dos Quinze leitos possuem respiradores microprocessados. O estudo foi aprovado pelo Comitê de Etica e Comitê científico do hospital em dezembro de 2005 sob o no 158.

A população foi composta pelos pacientes internados na Unidade de Terapia Intensiva do hospital, Que utilizavam o dispositivo ventilatório. No período da pesQuisa, a ocupação anual da UTI era de 14,5 pacientes/ dia, sendo Que diariamente havia em média 10 pacientes em uso de ventilação mecânica. Os critérios de inclusão dos pacientes, tanto no período prospectivo como para o período retrospectivo, foram: a) Ter dispositivo ventilatório invasivo - o paciente poderia utilizar tubo orotraqueal ou traqueostomia, independentemente do calibre ou tipo do dispositivo ventilatório, b) utilizar ventilação mecânica, c) Ter sofrido extubação acidental decorrente do cuidado de enfermagem. Foram excluídos pacientes Que sofreram extubação acidental por outro motivo Que não o cuidado de enfermagem. A população do estudo foi de 142 pacientes em uso de ventilação mecânica. Destes 72 tiveram seus dados colhidos retrospectivamente, e 70 pacientes prospectivamente.

A técnica usada foi a de levantamento de dados primários em prontuário. $\mathrm{O}$ instrumento utilizado foi um formulário dividido em duas partes: a primeira relacionada a intubação e a segunda relacionada a extubação acidental. As variáveis levantadas da população foram idade, sexo, data de intubação e tipo de dispositivo ventilatório.

As variáveis da extubação foram:data, a hora e o momento da ocorrência destas extubações (banho no leito, transporte, mobilização e troca de fixação). Com esta parte do instrumento, foi possível determinar Quantos dias de ventilação mecânica o paciente tinha Quando ocorreu a extubação e, em Que momento do cuidado deu-se o ocorrido.

A coleta de dados compreendeu dois períodos a partir de um corte considerando a aprovação do Comitê de Ética em Pesquisa (CEP)em dezembro de 2005. A partir deste momento os prontuários foram separados em retrospectivos e prospectivos e organizados pela data de intubação em ordem crescente. Fez-se um levantamento dos seis meses anteriores à aprovação pelo CEP (julho a dezembro de 2005) para ver os pacientes Que se extubaram. Foi implantado o guia de prevenção da extubação e durante seis meses avaliado o resultado desta aplicação (fevereiro a agosto de 2006). Para efeito de padronizar e uniformizar a caracterização e registro, a extubação acidental foi definida como o deslocamento do dispositivo ventilatório Que gerasse necessidade de reposicionamento, podendo ou não ocasionar complicação ou dificuldade respiratória imediata ao paciente.

Os dados foram armazenados, utilizando-se o programa Excel, com aplicação da técnica de dupla digitação com vistas a evitar erros de transcrição. Os dados foram submetidos a estatística descritiva e cálculo da incidência da extubação, não sendo possível aplicação do teste do Qui-Quadrado pois teve-se freeüências menores de cinco.

\section{RESULTADOS E DISCUSSÃO}

Quanto ao sexo, no período retrospectivo foram $38(52,78 \%)$ e $34(47,22 \%)$ do sexo masculino e feminino respectivamente e no 
prospectivo haviam $33(47,14 \%)$ e $37(52,86 \%)$ do sexo masculino e feminino.

Quanto à idade, predominaram os pacientes entre 71 a 90 anos (62\%) em ambos os períodos. Em relação ao dispositivo ventilatório, $52,78 \%$ (antes) e $47,14 \%$ (depois) dos pacientes usaram tubo traeueal. Os restantes em cada período foram de pacientes Que usaram traQueostomia.

O tempo de ventilação mecânica foi medido em dias. O total encontrado foi de 3.77I dias de ventilação mecânica, sendo 1830 dias na fase retrospectiva e 1941 na prospectiva. A média em dias de ventilação mecânica Que cada paciente no período retrospectivo foi de 25,4 e no prospectivo foi de 27,7 dias.

Os dados encontrados mostram Que, o número de pacientes do período retrospectivo e prospectivo foi praticamente o mesmo; a divisão por sexo foi Quase Que idêntica e, a idade da população foi concentrada na mesma faixa etária nos dois grupos.

\section{A incidência da extubação acidental}

No Quadro I está apresentado o número total de dias em ventilação; a incidência de extubação por 1.000 dias de ventilação e o percentual de extubação para cada grupo.

Antes da implementação do guia a taxa foi de $8,33 \%$ extubações acidentais ocasionadas pela equipe de enfermagem reduzindo para $2,85 \%$ eventos.Em relação às taxas de extubações acidentais encontradas, na revisão feita por Yeh et $\mathrm{al}^{(1)}$, estudos de 1994 a 2002 indicam Que a incidência de extubação não planejada varia de $3 \%$ a $14 \%{ }^{(4,6-12)}$. Destes casos de extubação não planejada, 77.9 a $87 \%$ foram auto-extubações e 13 a 22,1\% foram acidentais ${ }^{(7,8)}$. Outro estudo $^{(13)}$, porém, ressalta Que a incidência de extubação não planejada varia de 2,8\% a 20.6\% em diferentes investigações. Esta percentagem depende amplamente das características das Unidades de Terapia Intensiva e da duração da ventilação artificial.

Em termos de incidência houve 3,27 eventos e 1,03 respectivamente no período retrospectivo e prospectivo. Os trabalhos correlatos apresentam incidências Que oscilam de 16,4 eventos $^{(2)}$ a $3,85^{(3)}$. Essas incidências são para QualQuer tipo de extubação acidental o que não é o caso desta pesquisa que apenas mensura as extubações acidentais relacionadas com o cuidado de enfermagem.

No Quadro I também está apresntado o cuidado de enfermagem em Que ocorreu a extubação.

\section{A troca de fixação e a extubação acidental}

$\mathrm{Na}$ instituição onde esta pesQuisa foi realizada a assistência ventilatória ao paciente e os cuidados com o respirador são de responsabilidade do enfermeiro. São de competência do enfermeiro: a troca da fixação do dispositivo ventilatório; a verificação da pressão do cuff; a avaliação da patência do dispositivo ventilatório; a avaliação do posicionamento do dispositivo com o RX e, a monitorização com oximetria e capnografia.

A fixação do dispositivo ventilatório Quer seja tubo endotraqueal ou traqueostomia, é muito importante para a sua estabilidade, além de estar relacionada com a diminuição de lesões traqueais, também, evita desposicionamento, diminuindo a incidência de extubação acidental $^{(6)}$. Nesse sentido, manter o circuito do respirador apoiado em um suporte apropriado, reduz movimentos com o dispositivo de ventilação e este cuidado é fundamental para não gerar lesões por tração ${ }^{(14,15)}$.

Os enfermeiros, durante a troca de fixação também realizam a mensuração do cuff ou balonete. Neste procedimento o aparelho próprio vai ser acoplado ao balonete e o enfermeiro deve atentar para possíveis trações do dispositivo, uma vez Que o movimento da mão do enfermeiro e o peso do aparelho são fatores de risco para a extubação acidental.

A troca da fixação de uma traqueotomia sem cuff, pode representar maior facilidade para extubação acidental. Recomendase Que, em cânulas mais curtas, (como as sem cuff), não se utilize curativos grandes para não obstruir a visão completa da traeueostomia ${ }^{(15)}$.

Alguns sinais podem representar extubação acidental como: a dificuldade de progredir a sonda e o esforço respiratório. O enfermeiro deve saber reconhecer e diferenciar Quando se trata de extubação acidental e Quando é uma obstrução do dispositivo ventilatório.

Sinais Que indicam Que o tubo não está patente são: aumento da pressão de pico (pressão máxima) e diminuição da complacência pulmonar; dificuldade em progredir a sonda de aspiração traQueal (é um importante sinal); Quando o paciente é ventilado manualmente não pode haver resistência e, Quando ventilando espontaneamente, não apresentar esforço ${ }^{(16)}$.

A ausculta pulmonar é outro recurso utilizado para a confirmação da posição do tubo traQueal, além do controle da numeração do

\begin{tabular}{|lcc|}
\hline MEDIDAS & RETROSPECTIVO (72) & PROSPECTIVO (70) \\
\hline Dias de ventilação & 1830 & 1941 \\
Incidência da extubação & 3,2786 & 1,0303 \\
Percentual de extubações & $8,33 \%$ & $2,85 \%$ \\
Número de extubações & 6 & 2 \\
\hline CUIDADOS DE ENFERMAGEM & RETROSPECTIVO(6) & PROSPECTIVO(2) \\
\hline Momentos da extubação & 2 & - \\
Troca da fixação & 1 & - \\
Transporte & 2 & 1 \\
Mudança de decúbito & 1 & 1 \\
Banho no leito & 6 & 2 \\
\hline TOTAL & 6 & \\
\hline
\end{tabular}

Quadro 1. Resultados da extubação acidental e cuidados de enfermagem. Rio de Janeiro, 2006. 
dispositivo e do acompanhamento radiológico. Todos são parâmetros devem ser monitorados pelo enfermeiro para detecção precoce de alterações no paciente, decorrentes da troca da fixação do dispositivo ventilatório.

Outro recurso que pode guiar o enfermeiro é a capnografia Que apresenta alteração imediata, no caso de uma extubação, enQuanto Que mudanças na oximetria é são lentas, principalmente, Quando o paciente tem estímulo respiratório e uma maior reserva de oxigênio ${ }^{(16,17)}$.

A enfermagem deve compreender Que ao se deparar com ausência de curva no capnógrafo, deve checar as conexões do circuito, pois a hipótese mais provável é desconexão do respirador, ou extubação acidental ${ }^{(16)}$.

Se o tipo de fixação for fita, esparadrapo ou tecido o protocolo recomenda Que seja utilizada uma tesoura para cortar o fixador do tubo traqueal, já que o uso da tesoura torna o procedimento mais seguro.

Entretanto, uma revisão sistemática mostrou Que não há um único método de estabilização do tubo endotraqueal Que possa ser considerado como superior, na minimização do deslocamento do tubo ou na ocorrência de extubação acidental ou não planejada ${ }^{(17)}$.

\section{O transporte e a extubação acidenta}

Em função da gravidade dos pacientes internados na Unidade de Terapia Intensiva, com freqüência é necessária a realização de exames de alta complexidade, Que tornam necessário o seu transporte. Assim os transportes internos ocorrem Quando da necessidade de realização de tomografia, ressonância magnética, cintilografia, hemodinâmica e encaminhamento a outros setores do hospital.

Recomenda-se Que o transporte seja acompanhado por um técnico e um enfermeiro e na ocorrência de uma extubação acidental, ela é considerada responsável pelo evento. Na maioria das vezes, o paciente está mais sedado, portanto, com menor estimulo respiratório espontâneo e menor reserva de oxigênio. Neste momento, ocorrem alterações hemodinâmicas e ventilatórias Que devem ser identificadas pelo enfermeiro envolvido no transporte e por sua vez, resolvidas ${ }^{(18)}$.

As alterações ventilatórias ocorrem, principalmente, pela necessidade da troca do respirador do leito pelo respirador de transporte, Que normalmente tem um menor recurso tecnológico. Este fato gera uma dificuldade de adaptação do paciente, Que por várias vezes necessita do uso de sedativos. Em alguns pacientes estas alterações ventilatórias podem levar a instabilidade hemodinâmica ${ }^{(18)}$.

A mudança do paciente da cama para a maca é um momento de risco e devem ser tomados cuidados com a estabilidade do dispositivo ventilatório. Quanto menor for a mobilização da cabeça e maior o número de profissionais envolvidos, menor será o risco de uma extubação acidental.

Para Que o circuito do respirador não tracione o dispositivo ventilatório durante a movimentação da maca do tomógrafo e do aparelho de ressonância, é preciso Que o respirador fique posicionado próximo ao paciente. Nestes casos costuma ser útil a existência na sala de tomografia de uma mangueira de oxigênio com comprimento maior e para a sala de ressonância, o circuito do respirador também de maior comprimento. Com estas medidas os exames são feitos com segurança e sem o risco da extubação acidental $^{(18)}$

Outro aspecto Que deve ser reforçado na orientação da equipe é sobre a necessidade de checar a fixação do dispositivo antes do início do transporte, independentemente do local para onde este paciente seria encaminhado. A recomendação é de Que, como rotina, se troque o fixador do dispositivo ventillatório.

Também é importante considerar Que há pacientes Que podem necessitar de modos ou parâmetros ventilatórios Que podem não estar disponíveis na unidade de destino ou durante o transporte. Sob estas circunstâncias, é primordial Que sejam definidos modos alternativos de ventilação mecânica durante o transporte para assegurar a estabilidade do paciente ${ }^{(18)}$.

Um problema freqüente para a realização de exames de tomografia e ressonância magnética em pacientes sob ventilação mecânica é impedir que o circuito do ventilator tracione o dispositivo ventilatório durante a movimentação do paciente da maca para o tomógrafo(19).

\section{A mudança de decúbito e a extubação acidental}

A mudança de decúbito é um cuidado da equipe de enfermagem de grande importância para o paciente. Sua realização minimiza várias complicações associadas à ventilação mecânica de tal forma Que Quando a mudança de decúbito é correlacionada à ausculta pulmonar e avaliação radiológica, gera uma drenagem postural e com isso otimiza a expansão pulmonar ${ }^{(15,20)}$.

Um paciente acamado, como é o da Unidade de Terapia Intensiva, realiza mudanças de decúbito em sua maioria de duas em duas horas. Esse procedimento requer técnica, pois o paciente crítico não está sozinho no leito, está acompanhado de equipamentos Que o mantém vivo e caso ocorra desposicionamento de algum acessório, pode representar a morte ou atraso no tratamento.

Reforçando este aspecto, em sete estudos clínicos conduzidos entre 1995 e 2002 para determinar a freqüência e os fatores de risco associados com a extubação não planejada, observou-se associação entre a extubação e a posição do paciente no leito a partir do segundo survey. Dentre as posições no leito Que mais provocaram extubação destacaram-se as laterais e as com elevação da cabeça a $30^{\circ}$. A posição Que mais diminuiu o risco de extubação foi o posicionamento do paciente na cadeira, de $22 \%$ no primeiro survey para $0 \%$ nos seguintes, principalmente pela introdução de cadeiras especiais Que permitiram uma melhor estabilização do tubo ${ }^{(2)}$.

Para Que a mudança de decúbito seja segura, recomenda-se a utilização do traçado móvel, o Que facilita a mobilização evitando fricção da pele no lençol e diminuição da força desprendida pelos Que a realizam ${ }^{(22)}$.

Outra recomendação para garantir segurança durante a mudança de decúbito nos pacientes em uso de ventilação mecânica, é de Que seja feito com no mínimo dois profissionais de enfermagem, pois há necessidade de um profissional sempre direcionar sua atenção para a estabilidade do dispositivo ventilatório, evitando a extubação acidental ${ }^{(22)}$.

Dentro das UTI a rotina para as mudanças de decúbito, costuma solicitar Que todos os pacientes realizem na mesma hora o decúbito. Ou seja, horas pares ou horas ímpares, com vistas a facilitar o controle e organização do cuidado. Provavelmente para Que esta 
determinação possa ser cumprida, o procedimento muitas vezes é realizado rapidamente com menor cuidado podendo, assim, provocar a extubação acidental.

Talvez, como primeira medida, intercalar o horário dos decúbitos de forma que os profissionais de enfermagem tenham um menor número de mudanças de decúbito por hora, desprendendo mais atenção ao procedimento. Também, uma segunda recomendação seria o maior envolvimento do enfermeiro neste procedimento independente da estabilidade hemodinâmica ${ }^{(22)}$.

Talvez a redução da extubação acidental durante a mudança de decúbito dependa da alteração de rotinas e sua execução com maior cuidado e menor pressa.

\section{O banho no leito e a extubação acidental}

A realização do banho no leito costuma ser uma técnica de domínio da equipe de enfermagem. Ela é descrita por diversos autores $^{(15,22)}$ com pequenas variações. O objetivo é sempre proporcionar conforto, evitar infecções, preservar a integridade da pele. Para que o banho no leito do paciente crítico seja eficiente e eficaz, a realização em etapas, é recomendada.

Recomenda-se Que a técnica do banho seja em cinco etapas: higiene do couro cabeludo, higiene do rosto e boca, higiene da genitália, higiene das mãos e higiene do corpo. Esta divisão ocorre por inúmeras razões como, por exemplo: manter temperatura corpórea, preservar a privacidade do paciente e diminuir a ocorrência de eventos adversos, entre eles, a extubação acidental ${ }^{(22)}$.

A extubação pode ocorrer durante a lateralização do corpo e isso é explicado pela perda da centralização da cabeça, sendo Que a orientação junto à enfermagem é de Que, um técnico fique responsável por manter a cabeça em posição de segurança.

Saber executar a técnica do banho no leito é muito importante, pois um movimento errado com a cabeça de um paciente acamado, Quando este se encontra intubado, pode significar a extubação acidental.

Parece persistir, entre os profissionais de enfermagem, a idéia de que o banho no leito é um procedimento banal e às vezes até pouco meritório para o profissional que o executa, sendo realizado pelos técnicos de enfermagem auxiliados e supervisionados pelos enfermeiros, somente Quando trata-se de pacientes mais instáveis.

Cabe lembrar que no momento da troca de lençóis e higienização do dorso, a visualização do rosto e, conseqüentemente, do dispositivo ventilatório está dificultada sendo, então, necessário Que a verificação da fixação e a estabilidade do circuito do ventilador seja continuamente revista, enQuanto o paciente permanecer em decúbito lateral ${ }^{(23)}$.

Como a visualização do dispositivo ventilatório está prejudicada, a identificação de uma extubação acidental enQuanto o paciente está lateralizado, pode não ser visível imediatamente, contando-se para isso com o recurso dos alarmes.

Caso ocorra uma extubação durante o banho no leito o alarme será acionado apenas com a informação de ter havido a ocorrência de uma desconexão. Esta informação deve ser valorizada, o enfermeiro deve apurar as causas do disparo do alarme e nunca desabilitar o mesmo Quando este é acionado. O enfermeiro deve lembrar que o ventilador tende a alarmar mais durante o banho, pois durante este procedimento ocorre à presença de eventos normais como, por exemplo, taquipnéia ou tosse ${ }^{(24)}$.

Mudanças de comportamento em relação ao banho são necessárias abolindo-se antigas rotinas como, por exemplo, o técnico de enfermagem iniciar o banho no leito, sem uma avaliação prévia por parte do enfermeiro das condições clínicas e de utilização dos dispositivos pelo enfermeiro.

\section{CONCLUSÕES}

Embora a extubação acidental seja considerada um importante indicador de Qualidade do cuidado de enfermagem em terapias intensivas, tem sido pouco investigada em relação a sua associação com o cuidado de enfermagem e as medidas de barreira Que impeçam ou evitem este evento.

A despeito das limitações desta pesquisa, o emprego do protocolo de cuidados de enfermagem especificamente associado às variáveis transporte do paciente, mudança de decúbito, banho no leito e a troca de fixação, provocou uma Queda na taxa da extubação acidental.

Sob outro ângulo, estudos como estes, onde protocolos são testados podem auxiliar os profissionais de enfermagem a compreender o impacto dos mesmos na diminuição de eventos adversos, como e o caso da extubação acidental.

Um dos fatores Que podem estar relacionados com o fato de se ter presenciado uma extubação acidental relacionada à mudança de decúbito e ao banho no leito pode ser em função de a enfermagem realizar a mudança de decúbito e o banho no leito para todos os pacientes na mesma hora. Ou seja, horas pares ou horas ímpares, com vistas a facilitar o controle e organização do cuidado. Desta maneira, o procedimento muitas vezes é realizado rapidamente o Que pode facilitar a extubação acidental. Mudar esta rotina, pode fazer com Que os profissionais de enfermagem tenham um menor número de mudanças de decúbito e banhos por hora, desprendendo mais atenção e tempo ao procedimento.

Limitações importantes desse estudo estão relacionadas ao tempo de levantamento de dados, à necessidade de separar dentro do estudo pacientes que utilizam tubo traqueal e traqueostomia,e a pequena amostra Que o representa. Uma possibilidade de melhorar a Qualidade do estudo consiste em aumentar o tempo de acompanhamento dos pacientes, o Que vem sendo realizado numa segunda etapa da peseuisa.

Os atuais resultados não permitem estabelecer uma relação de associação entre o protocolo e a incidência da extubação, pois há Que se considerar outras aspectos como a Questão da organização do trabalho numa UTI e a carga de trabalho da enfermagem. Por outro lado, a pesquisa permite levantar hipóteses acerca dos investimentos Que devem ser feitos em termos de educação na enfermagem, a importância no desenvolvimento de protocolos de cuidado em unidades intensivas e mostra a influência do banho no leito e da mudança de decúbito na extubacao acidental. Pretende-se dar continuidade a este estudo com novas medidas, prazos e amostra maior, para comparação com os resultados aQui apresentados. 


\section{REFERÊNCIAS}

I. YakoY. Manual de procedimentos invasivo realizados no CTI Atuação das Enfermeiras. Rio de Janeiro: Medsi; 2000.

2. Silva LD, Pereira SRM, Mesquita AMF. Procedimentos de enfermagem: Semiotécnica para o cuidado. Rio de laneiro: Medsi; 2005.

3. Richmond AL, Jarog DL, Hanson VM.Unplanned extubation in adult critical care. Quality improvement and education payoff. Crit Care Nurse 2004; 24(1): 32-7.

4. Yeh SH, Lee LN, Ho TH, Chiang MC, Lin LW . Implications of nursing care in the occurrence and conseguencces of unplanned extubation in adult intensive care units. J Inten Nurs 2004; 4 I (3): 434-8.

5. Bouza C, Garcia E, Diaz M, Segovia E, Rodriguez I. Unplanned extubation in orally intubated medical patients in the intensive care unit: a prospective cohort study. Heart Lung 2007; 36(4): 270-6.

6. Boulain T. Unplanned extubations in the adult intensive care unit: a prospective multicenter study. Association des Réanimateurs du Centre-Ouest. Am I Respir Crit Care Med 1998; $157(4$ Pt I): 1131-7.

7. Betbesé AJ, Pérez M, Bak E, Rialp G, Mancebo I. A prospective study of unplanned endotracheal extubation in intensive care unit patients. Crit Care Med 1998; 26(7): 1180-6.

8. Chevron V, Menard IF, Richard IC, Girault C, Leroy J, Bonmarchand G (1998) Unplanned extubation: risk factors of development and predictive criteria for reintubation. Crit Care Med; 26: 1049-53.

9. Christie IM, Dethlefsen M, Cane RD. Unplanned endotracheal extubation in the intensive care unit. I Clin Anesth 1986; 8:289-93.

10. Maguire GP, DeLorenzo LJ, Moggio RA. Unplanned extubation in the Intensive Care Unit: a Quality-of-care concern. Crit Care Nursing Quart 1994; 40-7.

I I. Razek T, Gracias V, Sulliavan D, Braxton C, Gandhi R, Gupta R, $E T$ al. Assessing the need for reintubation: a prospective evaluation of unplanned endotracheal extubation. I Trauma: Injury, Infection Crit Care 2000; 48:466-9.
12. Tindol Ir GA, DiBenedetto RJ, Kosciuk L. Unplanned extubations. Chest 1994; 5(6): 1804-7.

13. Grap MI, Glass C, Lindamood MO. Factors related to unplanned extubation of endotracheal tubes. Critical Care Nurse 1995; 15(2): 57-65.

14. Chan PKO, Fischer S, Stewart TE, Hallett DC, Hynes-Gay P, Lapinsky SE, et al. Practicing evidence-based medicine: the design and implementation of a multidisciplinary team-driven extubation protocol. Crit Care 200 I; 5:349-54.

15. Urden L, Stacy K, Lough M. Thelan's Critical Care Nursing: Diagnosis and Management. $4^{\text {th }}$ ed. St Louis: Mosby Inc; 2002.

16. Birkett KM, Southerland KA, Leslie GD.Reporting unplanned extubation. I: Intensive Crit Care Nurs 2005; 2 I (2): 65-75.

17. Gardner, AH, Robert A. Henson R, Osborne S, Gardner GE. Best practice in stabilisation of oral endotracheal tubes: a systematic review. Australian Critical Care 2005; 18(4): 158-65.

18. Warren J, Fromm RE, Orr RA, Rotello LC, Horst HM. American College of Critical Care Medicine. Guidelines for the inter- and intrahospital transport of critically ill patients. Crit Care Med 2004; 32(I): 305-6.

19. Balon IA. Common factors of spontaneous self-extubation in a critical care setting. I Trauma Nurs 200 I; 7(3): 238-42.

20. Scales K, Pilsworth I. A practical guide to extubation. Nursing Standard 2007; 22(2): 44-8.

21. De Lassence A. Impact of unplanned extubation e reintubation after weaning on nosocomial pneumonia risk in the intensive care unit: a prospective multicenter study. Rev Anest 2002; 97(1): 556-9.

22. Moons P, Sels K, De Becker W, De Geest S, Ferdinande P. Development of a risk assessment tool for deliberate selfextubation in intensive care patients. Inten Care Med 2004; 30(7): 1348-55

23. Tobin MI. Principles and practice of mechanical ventilation. $2^{\text {nd }}$ ed. New York: Mcgraw-Hill; 2006.

24. Amato M. Princípios da ventilación mecánica. Madrid: Permanyer Publicaciones; 1998. 